\title{
Erratum: Self-Consistent Adiabatic Inspiral and Transition Motion [Phys. Rev. Lett. 126, 241106 (2021)]
}

\author{
Geoffrey Compère® and Lorenzo Küchler \\ (Received 22 November 2021; published 11 January 2022)
}

DOI: 10.1103/PhysRevLett.128.029901

An error occurred on Eq. (37) of this Letter. The expansion (35) can be used for the self-force $\eta^{-1} f^{r}$ which admits a finite value $\eta^{-1} f_{[0] *}^{r}$ at the last stable orbit. Equivalently, $f_{[0]}^{r}=0$ and the leading order self-force starts at order $O_{s}(\eta)$. Equations (38), (40), (45), (46), (48), (50), and (51) are then valid but with $\varepsilon_{*}=0, \zeta_{*}=0$. As a result $f_{(0)}^{r}=O_{\eta}\left(\tilde{\tau}_{*}-\tilde{\tau}\right)^{3 / 2}$ in Eq. (51). The inspiral and transition equations are further consistent with $f_{(0)}^{r}=0$. As a result, from Eq. (13), $\delta_{(0)}=0$. As a consequence, the adiabatic inspiral equations can be consistently simplified with $\delta_{(0)}=0$. In particular, Eq. (15) becomes trivial and Eq. (16) becomes the radial evolution equation. Moreover, Eq. (25) fixes $r_{(0), 1}^{*}$ in terms of $\kappa_{(0), 2}^{*}$.

In conclusion, the matching between the leading order transition motion and the leading order inspiral motion is performed with a geodesic quasicircular velocity in the slow timescale expansion at leading order and without $O\left(\eta^{0}\right)$ selfforce. The last sentence of the abstract should be omitted. In the introduction, the sentence "As we will demonstrate, consistency of the matching between the forced inspiral motion and the transition motion will lead to a leading-order radial self-force in the slow timescale expansion that is determined by the secular change of angular velocity required by radiation reaction" should be omitted. Below Eq. (8), the sentence "As we will discuss below, the consistent matching of the adiabatic inspiral with the transition motion will require a leading-order radial self-force $f_{(0)}^{r}(\tilde{\tau})$, see Eq. (51) below." should be changed to "As we will discuss below, the consistent matching of the adiabatic inspiral with the transition motion will be consistent with canceling the leading-order radial self-force $f_{(0)}^{r}(\tilde{\tau})$, see Eq. (51) below." The sentence "This leads to a leading-order radial self-force in the slow timescale expansion that was overlooked so far in the self-force formalism." of the conclusion should be omitted. There is no other change.

We gratefully thank Adam Pound for a useful correspondence. G. C. is Senior Research Associate of the Fonds de la Recherche Scientifique F.R.S.-FNRS (Belgium). L. K. acknowledges support from the European Spatial Agency Prodex experiment arrangement 4000129178 for the LISA gravitational wave observatory Cosmic Vision L3. 